\title{
Islanding Detection Based on S - Transform and Neural Network
}

\author{
Weishi Man, Zongyao Zhu, Zhiyu Zhang, Ruwei Ma, Jianhua Wang \\ Automation \& Information Engineering, Xi'an University of Technology, Beilin District, Jinhua \\ Road Number 5,Xi'an,Shanxi Province, China, \\ E-mail:mws_xaut@126.com
}

Keywords: Islanding Detection; S Transform; Neural Network; Nondetection Zone

\begin{abstract}
The passive methods have a large nondetection zone (DNZ) and a long detecting time. In addition, the threshold of the passive method is difficult to set. However, the active method will affect the power quality. So, an novel islanding detection method based on S - transform(ST) and neural network was proposed. In this method, ST was adopted to extract feature vector from the voltage of the point of common coupling (PCC), and then the feature vector is sent to the neural network after training to determine whether islanding occured. The simulation results show that the islanding can be detected accurately and quickly even when the power is perfectly matched, and in the presence of disturbances such as grid voltage fluctuation, harmonic disturbance, load switching, grid fault, etc. Avoid the problem of false tripping caused by various disturbances.
\end{abstract}

\section{Introduction}

With the development of society, the demand for energy is growing, distributed generation (DG) gradually plays an increasingly important role in the power system, but also brings a lot of problems, islanding detection is one of them [1]. Island state will be dangerous to the power system maintenance personnel and users, and it will cause some security risks for the equipment. Therefore, when the island occurs, you must quickly detect the island state and stop the DG supply to load.

At present, many methods have been proposed for island detection, which are mainly divided into three kinds: island detection methods based on communication, active methods and passive methods. The islanding detection methods based on communication [2] , which not only have no nondetection zone, but also can detect accurately and reliably. however the cost is high, and it can not be applied in the large-scale. Passive methods [3-4] does not affect the power quality, but the detection time is long and the nondetection zone is large. The active method [5-6] has a small nondetection zone and a fast detection speed. However, due to the introduction of the amount of disturbance, it affects the power quality and is prone to failure due to the dilution effect [7] in the case of multiple inverters.

With the popularity of distributed generation system and the increasing demand for power quality, it is of practical significance to study the pure passive method which has no effect on power quality. Literature [8] is an islanding detection method based on discrete wavelet, but the threshold is difficult to determine. In [9-10], wavelet and decision tree are used to detect the island, but the 
computational burden of the algorithm is greatly increased due to extract the feature quantity of the three-phase voltage and current signal at the same time. In [11], the wavelet is used to extract the characteristic values of the voltage and current signals, and then through the neural network for judgment. However, when the neural network is trained, the voltage fluctuation, harmonic disturbance and failure are not fully considered, which would cause misjudgment problem. In [12], the feature vector is extracted from the PCC point voltage by wavelet packet, and then the neural network and the support vector machine are used to detect the island. However, the above methods have the choice of mother wavelet. In [9] and [11] , dB4 wavelet is chosen as the mother wavelet. In [12], dB5 is chosen as the mother wavelet and [13] uses Biorthogonal 1.5 as the mother wavelet. In [14], a new wavelet is designed specifically for islanding detection, but this wavelet has not been verified by engineering. At present, there is no definite basis for the selection of mother wavelet. Most of them are based on experience and experiment, and different mother wavelet has a great influence on the analysis result.

$\mathrm{S}$ transform and wavelet transform are multi resolution analysis methods, but wavelet transform is not strictly in the sense of time-frequency analysis, it does not exactly correspond to the frequency, and S-transform can correspond to frequency [15]. And S transform is no problem of selection of mother wavelet compared with wavelet transform. Wavelet transforms extract low-band information layer by layer, but it can lose high-frequency information with large number of feature information, and S-transform can directly select the desired feature frequency band. In [16], the effect of S-transform and wavelet transform on islanding and power quality detection is compared. It is proved that the performance of S-transform is better than that of wavelet transform.

In order to solve the above problems, this paper proposes an islanding detection method based on S-transform and neural network. The S-transform is used to extract the characteristic quantity of the three-phase voltage of PCC. The neural network is used to determine whether or not the island has occurred and then send the trip signal.At the same time, we fully consider the influence of various disturbances on island detection under different power flow conditions, and avoid the problem of erroneous trip. By modeling and verifying on MATLAB, this method can accurately and quickly determine the island and non-island state in $20 \mathrm{~ms}$ under different load conditions and various disturbance conditions.

\section{S-Transform}

The S-transform is evolved from the combination of wavelet transform and short-time Fourier transform (STFT), first proposed by R.G.Stockwell et al. In [15]. The mathematical expression is as follows:

$$
S(\tau, v)=\int_{-\infty}^{+\infty} g(t) \omega(\tau-t, v) \mathrm{e}^{(-\mathrm{j} 2 \pi v t)} \mathrm{d} t
$$

In equation (1): $\tau$ and $v$ are the time and frequency of the $\mathrm{S}$ domain, $\omega(\tau-t, v)=\frac{|v|}{\sqrt{2 \pi}} \mathrm{e}^{-\frac{(\tau-t)^{2} v^{2}}{2}}$ is all in the unit area frequency $v$ of the generalized window function.

It can be seen from (1) that the advantage of S transform with respect to STFT is that the height and width of the Gaussian window vary with frequency. Which has a higher frequency resolution for the low frequency signal and has a higher temporal resolution for the high frequency signal. And the S-transform spectrum is directly related to the Fourier spectrum, maintaining the absolute phase information of the signal. 


\section{Proposed Island Detection Method}

\subsection{Detection method flow}

The equivalent impedance of the system observed by the DG side before and after the occurrence of the island is significantly changed, and the PCC point voltage will be affected by the PCC point harmonic current and the equivalent impedance [12], so when the island occurs that the PCC voltage will contain a large number of feature information, So this paper chooses the PCC point voltage signal sampling to carry out island detection.

In this paper, the islanding detection method was shown in Figure 1. Firstly, the three-phase voltage signal of the PCC point is sampled and the signal is transformed by ST. Then the feature vector is extracted according to the result of $\mathrm{S}$ transform. Finally, the neural network is used to judge whether the island has occurred.

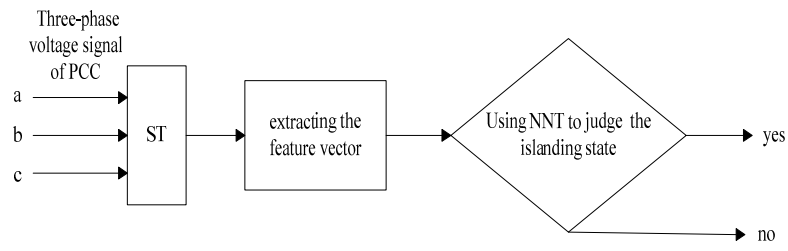

Figure 1 Flow chart of the detection method.

\subsection{Extraction of feature vector}

Considering the accuracy of the algorithm, the detection speed and the actual hardware implementation conditions, set the sampling frequency to $10 \mathrm{KHz}$. The figure 2 is the A phase voltage signal of PCC point when the island occured,. Figure 3 is its modulus time-frequency diagram after $\mathrm{S}$ transformation. The simulation time is $0.1 \mathrm{~s}$ and the island occured at the $0.06 \mathrm{~s}$.

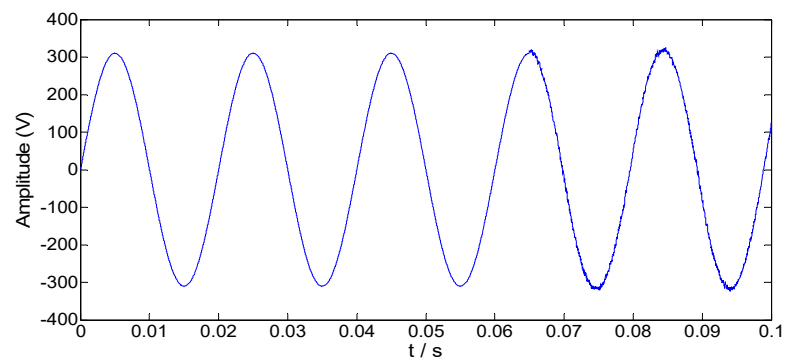

Figure 2 voltage waveform of PCC.

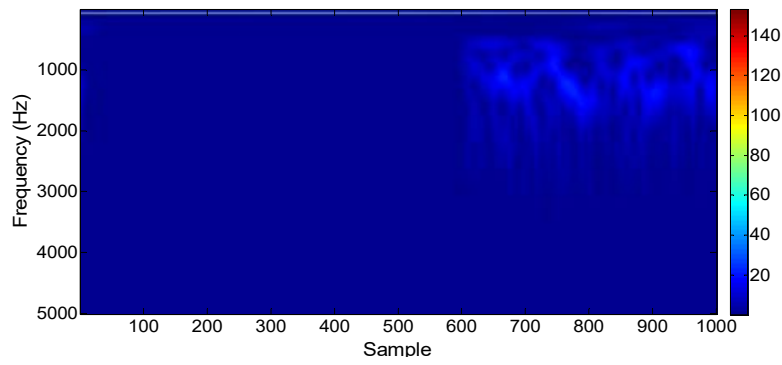

Figure 3 time-frequency diagram.

Since the sampling frequency is $10 \mathrm{KHz}$, according to the Nyquist sampling theorem, the vertical axis represents $0 \sim 5000 \mathrm{~Hz}$ frequency. It can be seen that most of the feature information is included in the frequency band of $250 \sim 3000 \mathrm{~Hz}$ when the island is occured. In many cases, all the 
simulation results can satisfy the feature.So take this band for feature frequency band.

The three-phase voltage signals of PCC are respectively taken by S transform, and the selected frequency bands in one cycle are calculated and extracted, and the following four feature quantities are extracted:

(1) the sum of the infinite norm of the three phases;

(2) the sum of the three-phase average;

(3) the sum of the three-phase mean square error;

(4) the sum of the three-phase maximum.

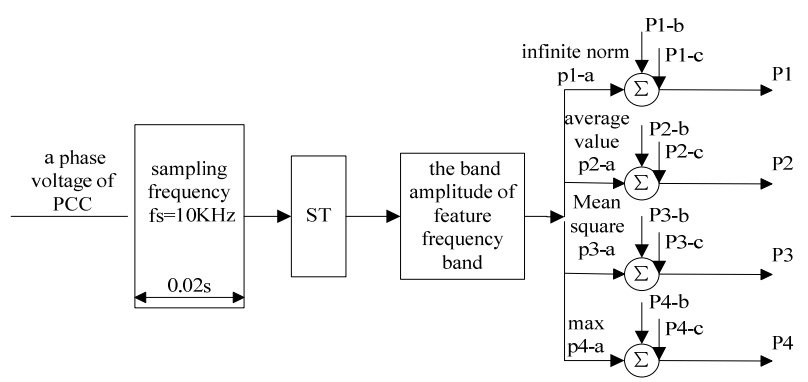

Figure 4 The flow chart of feature quantity extraction.

Figure 4 is the extracted method of feature quantity, the calculated four feature quantities constitute the input feature vector of the neural network. In order to simplify the flow chart, which only shows processing of the a-phase voltage, the processing of the b-phase and the c-phase is the same as that of the a-phase.

\subsection{Neural network structure}

Neural network unique non-linear adaptive information processing capabilities to the pattern recognition, intelligent control, prediction and other fields have been successfully applied. The use of its powerful system identification capabilities can solve the difficult problem of the threshold setting in the traditional passive islanding detection methods. BP network is a multi-layer feedforward neural network. At present, in the practical application of artificial neural network, the vast majority uses the BP network and its change form.

However, BP network has some problems such as slow convergence and local minima, in order to overcome the shortcomings of BP algorithm, this paper adopts the quasi-Newton algorithm.which is the improved algorithm of BP algorithm. The network structure includes input layer, hidden layer and output layer. Transfer function using logsig function, because the extracted feature vector is four-dimensional, the input node is set to four. And the output only island and non-island two states, so the output node is set to 1 . The number of hidden nodes is chosen according to the empirical formula (2). Finally, after repeated simulation experiments to determine the number of hidden layer nodes is 10 , this time training speed and recognition accuracy have achieved very good results.

$$
n=\sqrt{n_{i+} n_{o}}+a
$$

In the formula (2), $n$ is the number of hidden nodes. $n_{i}$ is the number of input nodes, $n_{o}$ is the number of output nodes, and $a$ is any constant between 1 and 10 . 


\section{Experimental Simulation}

\subsection{Simulation system model}

Simulation model shown in figure 5, the distributed generation system using photovoltaic power generation system, the large power grid uses the source with programmable three-phase voltage.

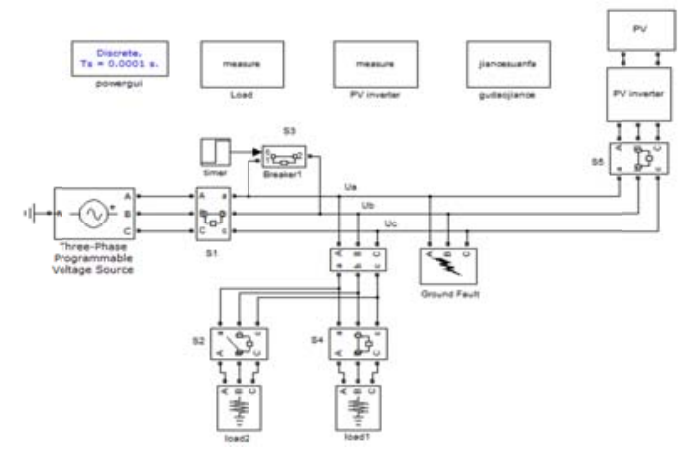

Figure 5 Simulation model diagram.

In this paper, the effects of load mutation, grid voltage mutation, harmonic disturbance, single phase to ground fault (AG), phase short circuit (AB), two-phase ground fault (ABG), three-phase ground fault $(\mathrm{ABCG})$ and so on to the islanding detection are simulated.

\subsection{Training of neural network}

The distributed generation (DG) system can be run in three different modes:

(1) the power required for the load is greater than DG, the grid and DG supply to the load at the same time;

(2) the load and DG power exactly match, only DG power supply to the load;

(3) DG power is greater than the load required power, the load is fully supplied by the DG, and DG supply power to large power grid at the same time.

In this paper, we set different load values in these three modes to simulate the normal connection, the island and various disturbance mentioned in Section A. A total of 104 sets of data were simulated for neural network training, and 110 sets of data (including 50 islands and 60 non islands) were simulated to test the performance of the method described in this paper.

\section{Simulation Result and Analysis}

The simulation waveform is shown below. It shows only the island state where the DG output power is very close to that of the load. The waveforms of the four cases including island, the grid voltage drop, $\mathrm{AG}$ and load mutation are shown below. The load parameters are set to: $\mathrm{P}=23 \mathrm{KW}$, $\mathrm{QL}=\mathrm{QC}=0$, and the power mismatch rate is only $3 \%$. 


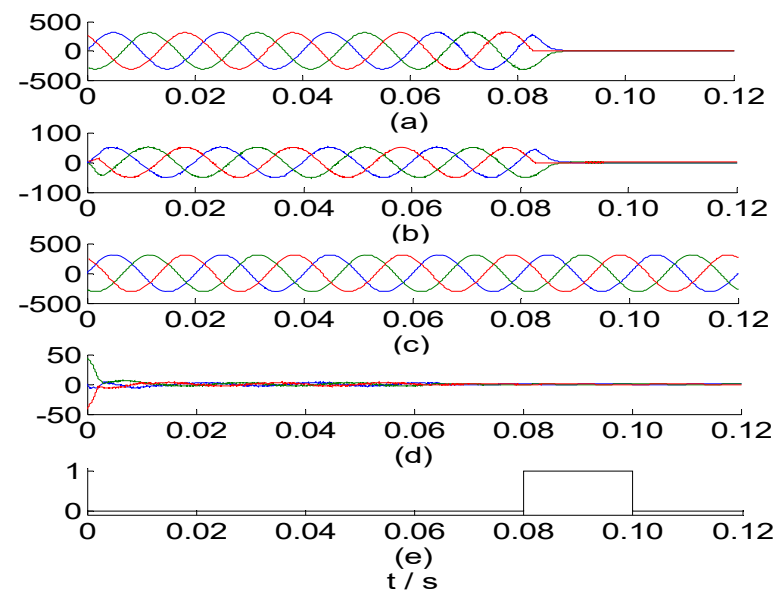

Figure 6 the waveform when the island occurs.

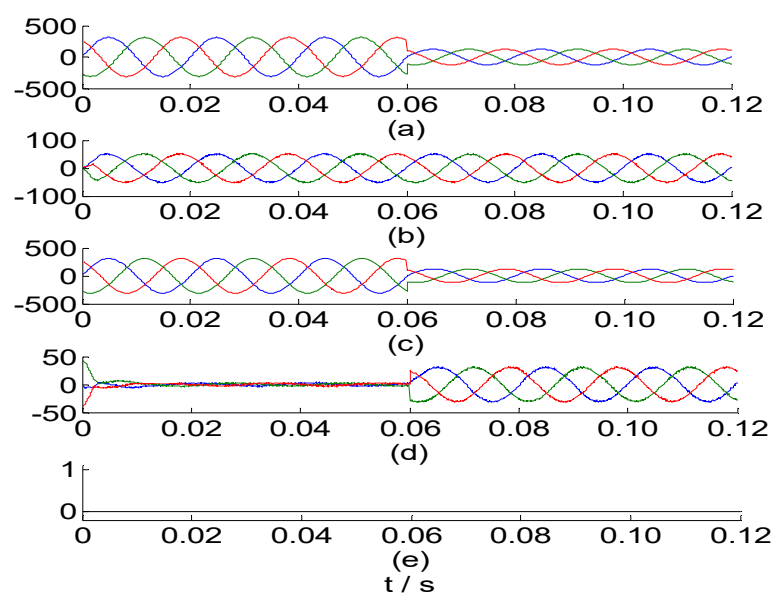

Figure 7 the waveform when the voltage changes.

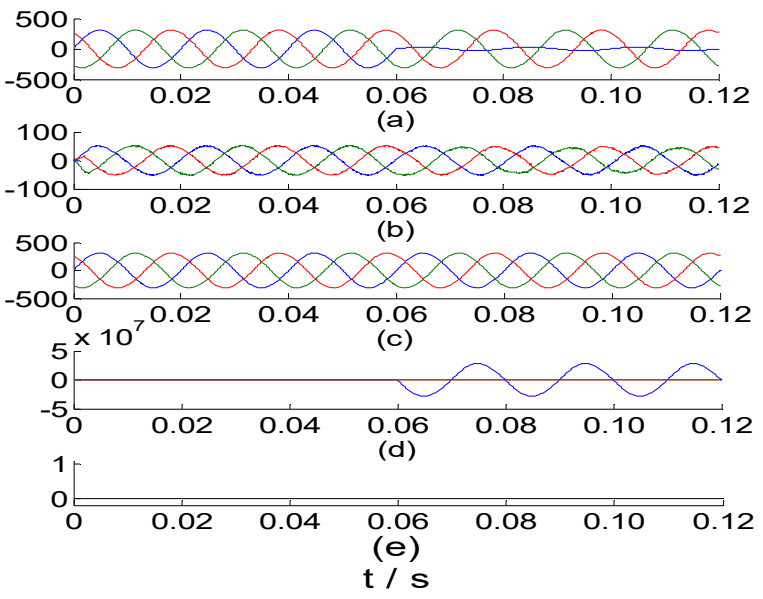

Figure 8 the waveform when AG. 


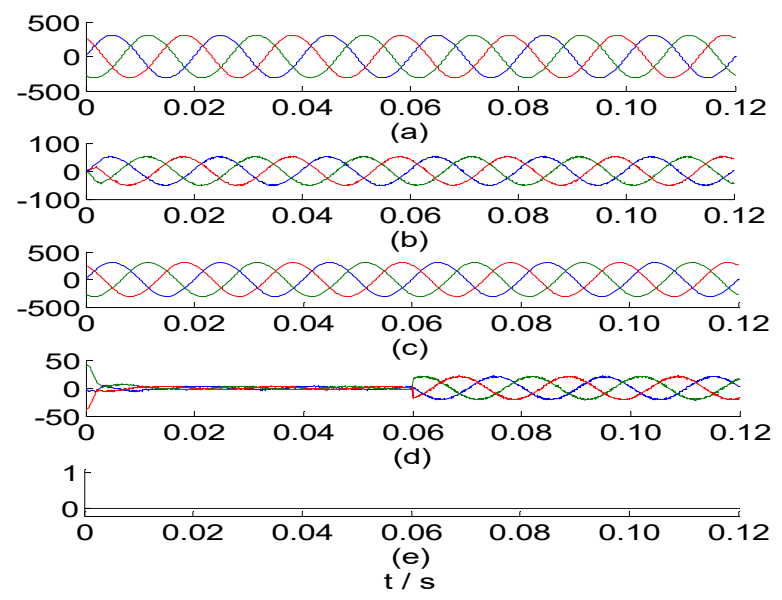

Figure 9 the waveform when the load changes.

Figure 6 shows the waveform after island occurs when circuit breaker is off in the $0.06 \mathrm{~s}$. (a)-(d) show the output of PPC voltage, inverter current, grid voltage, grid current and neural network output, respectively .due to load and DG power mismatch is very small, so the island before the grid current is very small, the island after the PCC point voltage amplitude has not changed, The grid current drops to zero. After a cycle of islanding, the neural network detects the island state to issue a trip signal to the inverter. The PCC voltage and the inverter current are reduced to zero and the detection time is only $0.02 \mathrm{~s}$.

Figure 7 shows $0.06 \mathrm{~s}$ when the grid voltage drop $60 \%$, the grid current with the rapid rise, PCC point voltage drops rapidly, but it is non-island state, so the neural network output is still zero, did not send the trip signal.

Figure 8 shows the waveform when the single-phase ground is short when it is $0.06 \mathrm{~s}$. It can be seen that a phase voltage drops close to 0 in the PCC point when a phase grounding short circuit occurs, the a-phase current of the power grid increases rapidly. But it is non-island state, so the neural network continues to output zero, the detection is correct.

Figure 9 shows the waveform of local load increase, when the load increases after the DG provided by the power can not meet the needs of the load, so the grid current to the load transmission power. But this time also belong to the non-island state, so the neural network to continue to output zero, the detection is correct.

Through the detection of the test samples, it is proved that this method can detect the island state accurately and quickly in various power flow and various disturbances. The algorithm detects only one power cycle of $0.02 \mathrm{~s}$, fully satisfying IEEE Standard 1547 [17] less than the detection time requirement of $2 \mathrm{~s}$.

\section{Conclusion}

In this paper, an island detection algorithm based on $\mathrm{S}$ transform and neural network is proposed. The time - frequency analysis ability of S - transform is used to extract the transient feature quantity of the island and various disturbances, and then the neural network is used to identify the island and the non - island state. A large number of simulation results show that the method described in this paper can accurately and quickly judge the island state even when the power mismatch is small and the existence of various disturbances, and it has no any effect on the power quality. 


\section{References}

[1] Cheng Qiming, Wang Yingfei, Cheng Yinman, Wang Mingmei. Overview study on islanding detecting methods for distributed generation grid-connected system[J]. Power System Protection and Control, 2011, 06:147-154.

[2] Horgan 5, Iannucci J, Whitaker C, Cibulka L, Erdman W. Assessment of the Nevada Test Site as a Site for Distributed Resource Testing and Project Plan. NREL/SR-560-31931, 2002.

[3] J. Merino, P. Mendoza-Araya, G. Venkataramanan and M. Baysal, "Islanding Detection in Microgrids Using Harmonic Signatures," in IEEE Transactions on Power Delivery, vol. 30, no. 5, pp. 2102-2109, Oct. 2015.

[4] Ma Jing, Michao, Wang Zengping. A novel islanding detection method based on positive feedback of voltage harmonic distortion[J]. Automation of Electric Power Systems, 2012(01).

[5] Bei Taizhou, Wang Ping, Cai Mengmeng. An islanding detection method with the third harmonic injection for distributed grid-connected PV inverters[J]. Transactions of China Electrotechnical Society, 2015,07:44-51.

[6] Jia Ke, Huan Kaixiang, Wei Hongsheng, Bi Tianshu, He Guoqing. An islanding detection method based on impedance estimation using external centralized disturbances for multi-distributed generation system[J]. Transactions of China Electrotechnical Society,2016,13:120-129.

[7] Xie Dong, Zhang Xing. Analysis of dilution phenomenon for active frequency shift islanding detection method[J]. Power System Protection and Control,2013,04:84-90.

[8] He Meimei, Li Huaqiang, Chen Jing, Wei Hongfen, Lin Maojun. Islanding detection based on discrete wavelet transform for distributed generation[J]. Electric Power Automation Equipment,2012,10:103-108.

[9] L. W. Arachchige and A. Rajapakse, "A pattern recognition approach for detecting power islands using transient signals - Part I: Design and implementation," 2011 IEEE Power and Energy Society General Meeting, San Diego, CA, 2011, pp. 1-1.

[10] N. W. A. Lidula and A. D. Rajapakse, "A Pattern-Recognition Approach for Detecting Power Islands Using Transient Signals_-Part II: Performance Evaluation," in IEEE Transactions on Power Delivery, vol. 27, no. 3, pp. 1071-1080, July 2012.

[11] M. S. ElNozahy, E. F. El-Saadany and M. M. A. Salama, "A robust wavelet-ANN based technique for islanding detection," 2011 IEEE Power and Energy Society General Meeting, San Diego, CA, 2011, pp. 1-8.

[12] H. T. Do, X. Zhang, N. V. Nguyen, S. S. Li and T. T. T. Chu, "Passive-Islanding Detection Method Using the Wavelet Packet Transform in Grid-Connected Photovoltaic Systems," in IEEE Transactions on Power Electronics, vol. 31, no. 10, pp. 6955-6967, Oct. 2016.

[13] A. Pigazo, M. Liserre, R. A. Mastromauro, V. M. Moreno and A. Dell'Aquila, "Wavelet-Based Islanding Detection in Grid-Connected PV Systems," in IEEE Transactions on Industrial Electronics, vol. 56, no. 11, pp. 4445-4455, Nov. 2009.

[14] S. Alshareef, S. Talwar and W. G. Morsi, "A New Approach Based on Wavelet Design and Machine Learning for Islanding Detection of Distributed Generation," in IEEE Transactions on Smart Grid, vol. 5, no. 4, pp. 1575-1583, July 2014.

[15] R. G. Stockwell, L. Mansinha and R. P. Lowe, "Localization of the complex spectrum: the S transform," in IEEE Transactions on Signal Processing, vol. 44, no. 4, pp. 998-1001, Apr 1996.

[16] P. K. Ray, N. Kishor and S. R. Mohanty, "Islanding and Power Quality Disturbance Detection in Grid-Connected Hybrid Power System Using Wavelet and S -Transform," inIEEE Transactions on Smart Grid, vol. 3, no. 3, pp. 1082-1094, Sept. 2012.

[17] IEEE Standard for Interconnecting Distributed Resources With Electric Power Systems, IEEE Standard 1547-2003, 2003, pp.1-6. 